\title{
A Practice-Based Evaluation of Distress Screening Protocol Adherence and Medical Service Utilization
}

\author{
Brad Zebrack, PhD, MSW, MPH', ; Karen Kayser, PhD, MSWc; Deborah Bybee, PhD; Lynne Padgett, PhD; \\ Laura Sundstrom, MSWa; Chad Jobin, MSW; ${ }^{\mathrm{a}}$; and Julianne Oktay, PhD, MSWf,g
}

\begin{abstract}
Background: This study examined the extent to which cancer programs demonstrated adherence to their own prescribed screening protocol, and whether adherence to that protocol was associated with medical service utilization. The hypothesis is that higher rates of service utilization are associated with lower rates of adherence to screening protocols. Methods: Oncology social workers at Commission on Cancer-accredited cancer programs reviewed electronic health records (EHRs) in their respective cancer programs during a 2-month period in 2014. Rates of overall adherence to a prescribed distress screening protocol were calculated based on documentation in the EHR that screening adherence and an appropriate clinical response had occurred. We examined documentation of emergency department (ED) use and hospitalization within 2 months after the screening visit. Results: Review of 8,409 EHRs across 55 cancer centers indicated that the overall adherence rate to screening protocols was $62.7 \%$. The highest rates of adherence were observed in Community Cancer Programs (76.3\%) and the lowest rates were in $\mathrm{NCl}$-designated Cancer Centers (43.3\%). Rates of medical service utilization were significantly higher than expected when overall protocol adherence was lacking. After controlling for patient and institutional characteristics, risk ratios for ED use (0.82) and hospitalization (0.81) suggest that when overall protocol adherence was documented, $18 \%$ to $19 \%$ fewer patients used these medical services. Conclusions: The observed associations between a mandated psychosocial care protocol and medical service utilization suggest opportunities for operational efficiencies and costs savings. Further investigations of protocol integrity, as well as the clinical care models by which psychosocial care is delivered, are warranted.
\end{abstract}

J Natl Compr Canc Netw 2017;15(7):903-912 doi:10.6004/jnccn.2017.0120

Since the 1970s, investigations of cancer-related psychological distress have consistently reported prevalence rates ranging between $20 \%$ and $40 \% \cdot{ }^{1-6}$ Distress, as defined by NCCN, is "a multifactorial unpleasant emotional experience of a psychological...social, and/ or spiritual nature that may interfere with the ability to cope effectively with cancer, its physical symptoms, and its treatment." 7 Distress is not a diagnosable disorder. Like fever or high blood pressure, distress is an indicator - a psychosocial marker-of conditions re-

From aUniversity of Michigan School of Social Work, and bUniversity of Michigan Comprehensive Cancer Center, Ann Arbor, Michigan; 'University of Louisville Kent School of Social Work, Louisville, Kentucky; ${ }^{\mathrm{d}}$ Michigan State University, Lansing, Michigan; 'American Cancer Society, Atlanta,

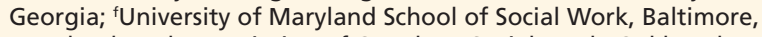

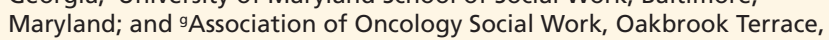
Illinois.

Submitted December 20, 2016; accepted for publication February 16, 2017. The authors have disclosed that they have no financial interests, arrangements, affiliations, or commercial interests with the manufacturers quiring further clinical evaluation and response, including redress of the distress itself. Elevated distress may suggest that depression or anxiety are present at clinically significant levels, or that a patient is struggling with co-occurring physical, psychological, social, or spiritual-existential challenges that are likely to disrupt adherence to therapy. ${ }^{8}$

Distressed patients tend to experience poorer health outcomes, greater mortality and morbidity, poorer immune function, and greater healthcare expenditures. ${ }^{9}$

of any products discussed in this article or their competitors. The design and conduct of this study were funded by the Association of Oncology Social Work.

Author contributions: Study conception and design: all authors. Acquisition of data: all authors. Analysis and interpretation of data: all authors. Drafting of manuscript: all authors.

Correspondence: Brad Zebrack, PhD, MSW, MPH, University of Michigan School of Social Work, 1080 South University, Ann Arbor, MI 48109-1106. E-mail: zebrack@umich.edu 
Distress also contributes to greater service use. ${ }^{10-12}$ In 2007, the Institute of Medicine (now Health and Medicine Division, National Academies of Sciences, Engineering, and Medicine) identified critical shortcomings in the delivery of psychosocial care for patients with cancer and their families, particularly with regard to the detection of psychological distress. ${ }^{13}$ In response, the American College of Surgeons' Commission on Cancer $(\mathrm{CoC})$, the primary accrediting body for as many as 1,500 cancer-treating programs serving approximately $70 \%$ of US patients with cancer, established new accreditation standards for patient-centered care, including Standard 3.2, a mandate requiring that all patients be screened for distress and that there be an appropriate clinical response when patients are identified as distressed. ${ }^{14}$

Evidence of the effect of distress screening on patient and institutional outcomes is mixed and underdeveloped. Some studies suggest that screening results in reductions in emotional distress, better quality of life, and improved patient-provider communication, along with increases in appropriate referral to psychosocial care providers. ${ }^{15,16}$ Others suggest that screening patients for distress may be of minimal benefit. ${ }^{17,18}$ Lacking, however, are studies demonstrating the effect of both screening and appropriate clinical response on patient and institutional outcomes. ${ }^{19,20}$ Just as we do not expect blood pressure screening alone to reduce symptoms of cardiovascular disease or stroke, we should not expect distress screening alone to improve outcomes. ${ }^{12}$ There must also be an appropriate clinical response when risk conditions are detected, because patient outcomes are improved after receipt of evidencebased psychosocial support interventions. ${ }^{21-25}$

Although now required as a component of quality psychosocial care, the CoC's distress screening standard does not prescribe a screening instrument nor dictate when or how often screening is to occur, other than at a "pivotal visit." The standard also does not dictate how providers must respond to distressed patients. Cancer programs determine their own protocols for clinical response (eg, immediate contact with a social worker, referral to supportive care services). Thus, given that individual cancer programs vary in their algorithms and pathways for how and when they screen for distress and respond to patients, the primary purpose of this study was to examine the capacity of individual cancer programs to adhere to their own prescribed distress screening protocols. It also examined the extent to which protocol adherence was associated with subsequent medical service utilization, specifically rates of emergency department (ED) use and hospitalization. We hypothesized that higher rates of service utilization would be associated with lower rates of adherence to screening protocols, after controlling for select patient and institutional factors.

\section{Methods}

This study was conducted under the auspices of the Association of Oncology Social Work (AOSW) Project to Assure Quality Cancer Care (APAQCC), a quality assurance/quality improvement project that evaluated capacity to deliver psychosocial care at CoC-accredited US cancer centers (or similar designation in Canada). Anonymized patient data from electronic health records (EHRs) were reviewed to evaluate program compliance with CoC Standard 3.2 and the extent to which compliance on a caseby-case basis was associated with ED use or hospitalization within 2 months after the patient visit at which screening was to occur, as per each institution's own protocol.

\section{Participants}

Details regarding the identification and selection of cancer programs for the APAQCC project are reported elsewhere. ${ }^{26}$ For purposes of this study, social workers at 55 participating cancer programs were charged with reviewing and abstracting data from EHRs at their respective institutions. All participating sites obtained Institutional Review Board approval or a waiver for nonregulated research activity prior to data collection.

\section{Data Collection}

From January through April 2015, social workers reviewed EHRs of patients seen in their respective cancer program during a 2-month window occurring between January 2014 and January 2015. For smaller cancer programs, data were feasibly collected for all patients; for larger programs, social workers were instructed to select all or a random subsample of records from single or multiple clinical units (eg, radiation oncology, medical oncology, multidisciplinary clinic). All social workers received formal 
Distress Screening Adherence

instructions (via webinar and a training manual) and ongoing guidance and support from the research staff on how to randomize select subsamples, if needed. Training materials also instructed social workers on how to abstract and code anonymized data (no personal health information was collected) and enter it into a Google form maintained by the principle investigator at the University of Michigan School of Social Work, where it was subsequently analyzed.

\section{Measures}

Screening Adherence, Responsiveness, and Overall Adherence: Assessing adherence to a prescribed protocol on a case-by-case basis involved locating documentation in an EHR that screening of the patient had occurred and that appropriate action was taken when clinically indicated. "Adherence" was noted as "yes" if an EHR contained a note, a scanned copy of the screening instrument, or a defined field with a score entered or a note indicating that screening had occurred. "Responsiveness" was noted as "yes" when the EHR contained documentation of appropriate action taken in response to the patient score, as per protocol (eg, referral, follow-up by social worker or other care provider, or no action when the screening score was below the program's defined cut-point for response). "Overall adherence" was noted as "yes" when documentation of screening adherence and responsiveness were confirmed.

Medical Service Utilization: Service utilization was indicated by EHR documentation that a patient had (1) visited the ED at the institution housing the cancer program; or (2) been hospitalized. Investigators tabulated these numbers when occurring within 2 months of a patient visit at which screening occurred or should have occurred according to protocol.

Patient and Institutional Characteristics: Each cancer program in this study was coded according to its CoC designation as an Academic Comprehensive Cancer Program, Comprehensive Community Cancer Program, Community Cancer Program (CCP), or NCI-designated Comprehensive Cancer Center Program (NCIP), which is based on type of facility, services provided, and number of analytic cases per year. ${ }^{14}$ Cancer program registries were queried for distribution of racial minority patients, with $40 \%$ distinguishing "minority institutions," similar to the NCl's criterion for defining a Minority-Based Com- munity Clinical Oncology Program. ${ }^{27}$ Data for determining patient age, race, sex, and cancer diagnosis were extracted from EHRs.

\section{Data Analysis}

Univariate statistics described patient and institutional characteristics, rates of adherence, responsiveness and overall adherence, and frequencies for ED use and hospitalization. Cross-tabulations and chi-square tests of statistical significance were calculated to examine variations in overall screening protocol adherence by patient and institutional characteristics, and to examine associations between overall adherence and medical service utilization. Mixed effects logistic regression was used to examine the effect of overall screening adherence on ED use and hospitalization rates, after controlling for patient and institutional characteristics. Analyses were conducted using HLM software with restricted maximum likelihood estimation. ${ }^{28}$ To enhance interpretation of data analytic results, odds ratios were converted to risk ratios using mixed-effects Poisson models with robust standard errors. ${ }^{29}$

Mixed effects with random intercept models account for random variations among cases nested within different institutions. Within-hospital sample sizes ranged from 10 to 467 , with 48 participating sites exceeding 40 cases. Statistical power analysis estimated a minimum detectable odds ratio of 0.80 with $80 \%$ power at 2 -tailed $P<.05$, given outcome base rates between $14 \%$ and $20 \%$ and screening adherence rates of $70 \%$ to $75 \%$. Intraclass coefficients (ICC) derived from these models estimated the proportion of variance in outcome that is attributed to variability among institutions composed of varied patient populations and varied screening procedures. An ICC of 0.15 (15\%) is considered an average proportion of variance due to random variations among cases nested within and across institutions. ${ }^{28}$

Prior to regression analysis, missing data patterns were examined. For the outcome variables, data were missing in 1,451 cases (17.3\%) for ED visits and 1,448 cases $(17.2 \%)$ for hospitalizations-rates well below those often reported in studies using EHRs. ${ }^{30,31}$ Our examination of these 2 outcome variables indicated that data were more likely to be missing in cases for which screening adherence was not documented; thus, outcome variables were not missing at random and could not reliably be imputed from existing data. ${ }^{32}$ There- 
Zebrack et al

\begin{tabular}{|c|c|c|c|c|c|}
\hline & $\begin{array}{c}\text { Total Cases } \\
(\%)\end{array}$ & $\begin{array}{l}\text { ACAD } \\
(\mathrm{N}=10)\end{array}$ & $\begin{array}{c}\text { CCCP } \\
(\mathrm{N}=24)\end{array}$ & $\begin{array}{c}\text { CCP } \\
(N=14)\end{array}$ & $\begin{array}{l}\text { NCIP } \\
(\mathrm{N}=7)\end{array}$ \\
\hline Cases, $\mathrm{n}$ & $8,409(100.0)$ & $1,742(20.7)$ & $3,054(36.3)$ & $1,479(17.6)$ & $2,134(25.4)$ \\
\hline \multicolumn{6}{|l|}{ Age, $y$} \\
\hline $15-39$ & $545(6.5)$ & $155(8.9)$ & $121(4.0)$ & $84(5.7)$ & $185(8.7)$ \\
\hline $40-64$ & $4,029(47.9)$ & $933(53.7)$ & $1,368(44.8)$ & $660(44.6)$ & $1,068(50.1)$ \\
\hline$\geq 65$ & $3,829(45.5)$ & $650(37.4)$ & $1,564(51.2)$ & $735(49.7)$ & $880(41.3)$ \\
\hline \multicolumn{6}{|l|}{ Race } \\
\hline White & $6,459(76.8)$ & 1,238 & $2,382(86.2)$ & $1,288(88.7)$ & $1,551(80.5)$ \\
\hline Black & $641(7.6)$ & $135(9.1)$ & $231(8.4)$ & $104(7.2)$ & $641(8.4)$ \\
\hline Hispanic/Latino & $295(3.5)$ & $76(5.1)$ & $64(2.3)$ & $39(2.7)$ & $116(6.0)$ \\
\hline Asian & $195(2.3)$ & $32(2.2)$ & $75(2.7)$ & $14(1.0)$ & $195(2.6)$ \\
\hline $\mathrm{Al} / \mathrm{AN} / \mathrm{NH} / \mathrm{PI}$ & $35(0.4)$ & $3(0.2)$ & $10(0.4)$ & $7(0.5)$ & $35(0.5)$ \\
\hline \multicolumn{6}{|l|}{ Sex } \\
\hline Female & $5,214(62.0)$ & $1,090(62.6)$ & $1,882(61.6)$ & $1,053(71.2)$ & $1,189(55.7)$ \\
\hline Male & $3,194(38.0)$ & $652(37.4)$ & $1,171(38.4)$ & $426(28.8)$ & $945(44.3)$ \\
\hline \multicolumn{6}{|l|}{ Cancer type } \\
\hline Brain/CNS & $131(1.6)$ & $30(1.7)$ & $46(1.5)$ & $15(1.0)$ & $40(1.9)$ \\
\hline Hodgkin lymphoma & $59(0.7)$ & $15(0.9)$ & $21(0.7)$ & $12(0.8)$ & $11(0.5)$ \\
\hline $\mathrm{NHL}$ & $353(4.2)$ & $59(3.4)$ & $142(4.6)$ & $79(5.3)$ & $73(3.4)$ \\
\hline Leukemia & $276(3.3)$ & $33(1.9)$ & $54(1.8)$ & $136(9.2)$ & $53(2.5)$ \\
\hline Melanoma & $261(3.1)$ & $29(1.7)$ & $57(1.9)$ & $13(0.9)$ & $162(7.6)$ \\
\hline Colorectal & $582(6.9)$ & $115(6.6)$ & $251(8.2)$ & $74(5.0)$ & $142(6.7)$ \\
\hline All other gastrointestinal & $722(8.6)$ & $142(8.2)$ & $255(8.3)$ & $72(4.9)$ & $253(11.9)$ \\
\hline Ovarian & $519(6.2)$ & $163(9.4)$ & $75(2.5)$ & $186(12.6)$ & $95(4.5)$ \\
\hline Prostate & $572(6.8)$ & $82(4.7)$ & $270(8.8)$ & $52(3.5)$ & $168(7.9)$ \\
\hline All other genitourinary & $886(10.5)$ & $142(8.2)$ & $187(6.1)$ & $281(19.0)$ & $286(13.4)$ \\
\hline Head and neck & $467(5.6)$ & $158(9.1)$ & 119 (3.9) & $53(3.6)$ & $137(6.4)$ \\
\hline Lung & $1,009(12.0)$ & $227(13.0)$ & $458(15.0)$ & $127(8.6)$ & $197(9.2)$ \\
\hline Breast (malignant) & $2,275(27.1)$ & $451(25.9)$ & $1,041(34.1)$ & $355(24.0)$ & $428(20.1)$ \\
\hline All others & 297 (3.5) & $106(6.1)$ & $78(2.6)$ & $24(1.6)$ & $89(4.2)$ \\
\hline
\end{tabular}

Percentages do not always total $100 \%$ due to missing data or lack of specification.

Abbreviations: ACAD, Academic Comprehensive Cancer Program; Al/AN/NH/PI, American Indian/Alaskan Native/Native Hawaiian/Pacific Islander; CCCP, Comprehensive Community Cancer Program; CCP, Community Cancer Program; CNS, central nervous system; NCIP, NCl-designated

Comprehensive Cancer Center Program NHL; non-Hodgkin's lymphoma.

fore, cases with missing outcome data were omitted from the analysis, and the sample sizes for each regression model vary. Missing data for explanatory independent variables was minimal, affecting just $5.3 \%$ of cases and $0.7 \%$ of the data matrix. To minimize case exclusion, missing values for explanatory variables were estimated using expectation maximization.

\section{Results}

Data were abstracted from EHRs of 8,409 patients confirmed as having cancer across 55 cancer centers.
Eleven cancer programs (20\%) met NCI criterion for being at an institution treating a significant proportion of racial minority patients; $18 \%$ of all cases $(n=1,541)$ were derived from minority institutions. Characteristics of participating cancer programs and patients are summarized in Table 1 .

\section{Adherence and Responsiveness to the Screening Protocol}

In $84 \%$ of participating cancer programs, patients were screened using the NCCN Distress Thermometer and Problem List or some modification thereof. 
Distress Screening Adherence

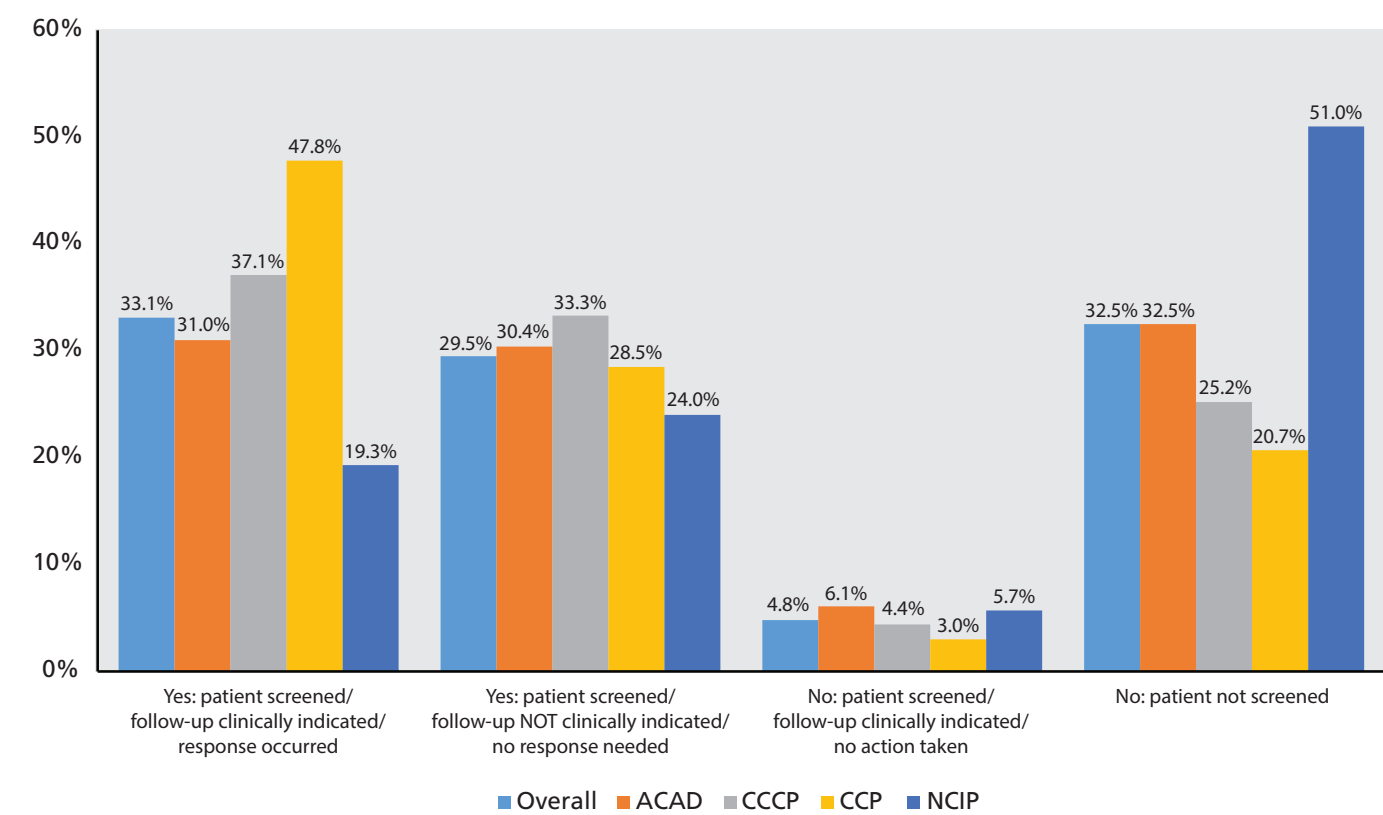

Figure 1. Documentation of appropriate clinical response to screening: was appropriate action taken? $(n=8,409)$ Cases screened $(n=5,685)$

Abbreviations: ACAD, Academic Comprehensive Cancer Program; CCCP, Comprehensive Community Cancer Program; CCP, Community Cancer Program; NCIP, NCl-designated Comprehensive Cancer Center Program.

Of 8,409 EHRs reviewed, 5,685 patients (67.6\%) were identified as being screened in accordance with each respective institution's protocol; 2,400 (28.5\%) were not. An additional 324 patients (3.9\%) declined to be screened and were re-coded as "patient not screened" for subsequent analyses.

Among the 5,685 patients screened, a clinical response was documented in 2,783 cases $(49.0 \%)$ in which screenings indicated need for response as per protocol. Clinical response was not clinically indicated (and thus not appropriate) in $43.5 \%$ of cases $(n=2,478)$. Documentation was absent in 424 cases $(7.5 \%)$ in which screens indicated need for a clinical response. The rate of responsiveness among patients when screened was $92.5 \%$. However, screening did not occur in 2,724 of the total 8,409 cases (32.4\%); therefore, the overall adherence rate for the entire sample of 8,409 cases was $62.6 \%$.

Overall adherence rates-the extent to which programs screened and responded appropriatelyvaried by institution and patient characteristics (Figure 1, Table 2). They were highest in CCPs (76.3\%) and lowest in NCIPs (43.3\%). Adherence rates for patients aged 15 to 39 years $(58.5 \%)$ were significantly lower than expected compared with older patients.
Highest rates of overall adherence were observed for patients identified in the EHR as Black/African American (70.5\%), and lowest for patients of American Indian/Alaska Native/Native Hawaiian/Pacific Island descent (45.7\%). Rates also varied significantly for patients depending on their cancer diagnosis (Table 2).

\section{Medical Service Utilization}

Among cases for which service utilization data were collected, 954 (13.7\%) used the ED at the institution where they were screened at least once, and 1,398 (20.1\%) were hospitalized at least once during the 2 months following the visit at which they were screened (Figure 2).

\section{Service Utilization and Overall Protocol Adherence}

Rates of ED use and hospitalization were significantly higher than expected when overall adherence was lacking. When overall protocol was followed, 12.8\% of patients were reported to have used the ED compared with $15.7 \%$ of patients for whom protocol was not followed $\left(X^{2}=9.97 ; P=.002\right)$. Among patients for whom overall protocol was followed, $18.6 \%$ were 
Zebrack et al

Table 2. Does Overall Adherence to a Distress Screening Protocol Vary by Institutional and Patient Characteristics?

\begin{tabular}{|c|c|c|c|}
\hline & No & Yes & $\begin{array}{c}X^{2} \\
\text { ( } P \text { Value })\end{array}$ \\
\hline \multicolumn{4}{|l|}{ Institution type } \\
\hline ACAD & $671(38.6)$ & $1,069(61.4)$ & \multirow{4}{*}{$\begin{array}{c}537.72 \\
(.000)\end{array}$} \\
\hline CCCP & $903(29.6)$ & $2,148(70.4)$ & \\
\hline CCP & $348(23.7)$ & $1,122(76.3)$ & \\
\hline NCIP & $1,207(56.7)$ & $922(43.3)$ & \\
\hline \multicolumn{4}{|l|}{ Minority institution } \\
\hline No & $2,553(37.2)$ & $4,301(62.8)$ & \multirow{2}{*}{$\begin{array}{c}.034 \\
(.854)\end{array}$} \\
\hline Yes, $\geq 40 \%$ racial minority patients & $576(37.5)$ & $960(62.5)$ & \\
\hline \multicolumn{4}{|l|}{ Age, $y$} \\
\hline $15-39$ & $226(41.5)$ & $318(58.5)$ & \multirow{3}{*}{$\begin{array}{c}6.16 \\
(.046)\end{array}$} \\
\hline $40-64$ & 1,459 (36.3) & $2,562(63.7)$ & \\
\hline$\geq 65$ & $1,438(37.7)$ & $2,381(62.3)$ & \\
\hline \multicolumn{4}{|l|}{ Race } \\
\hline White/Caucasian & $2,374(36.8)$ & $4,072(63.2)$ & \multirow{5}{*}{$\begin{array}{l}23.35 \\
(.000)\end{array}$} \\
\hline Black/African American & $188(29.5)$ & $449(70.5)$ & \\
\hline Hispanic/Latino & $116(39.3)$ & $179(60.7)$ & \\
\hline Asian & $84(43.3)$ & $110(56.7)$ & \\
\hline $\mathrm{Al} / \mathrm{AN} / \mathrm{NH} / \mathrm{PI}$ & $19(54.3)$ & $16(45.7)$ & \\
\hline \multicolumn{4}{|l|}{ Sex } \\
\hline Female & $1,907(36.7)$ & $3,294(63.3)$ & \multirow{2}{*}{$\begin{array}{c}2.34 \\
(.126)\end{array}$} \\
\hline Male & $1,222(38.3)$ & $1,966(61.7)$ & \\
\hline \multicolumn{3}{|l|}{ Cancer type } & \multirow{15}{*}{$\begin{array}{l}65.93 \\
(.000)\end{array}$} \\
\hline Breast (malignant) & $822(36.2)$ & $1,451(63.8)$ & \\
\hline Lung/Bronchus & $390(38.8)$ & $616(61.2)$ & \\
\hline Colorectal & $253(43.5)$ & $329(56.5)$ & \\
\hline All other gastrointestinal & $273(37.9)$ & $448(62.1)$ & \\
\hline Ovarian & $151(29.1)$ & $368(70.9)$ & \\
\hline Prostate & $192(33.6)$ & $379(66.4)$ & \\
\hline All other genitourinary & $298(33.8)$ & $584(66.2)$ & \\
\hline Head and neck & $173(37.0)$ & $294(63.0)$ & \\
\hline Hodgkin lymphoma & $19(32.8)$ & $39(67.2)$ & \\
\hline $\mathrm{NHL}$ & $125(35.8)$ & $224(64.2)$ & \\
\hline Leukemia & $116(42.0)$ & $160(58.0)$ & \\
\hline Melanoma & $125(48.4)$ & $133(51.6)$ & \\
\hline Brain/CNS & $50(38.2)$ & $81(61.8)$ & \\
\hline All other & $142(47.8)$ & $155(52.2)$ & \\
\hline
\end{tabular}

Subsample sizes vary due to missing data.

Abbreviations: ACAD, Academic Comprehensive Cancer Program; Al/AN/NH/PI, American Indian/Alaskan Native/Native Hawaiian/Pacific Islander; CCCP, Comprehensive Community Cancer Program; CCP, Community Cancer Program; CNS, central nervous system; NCIP, NCl-designated

Comprehensive Cancer Center Program; NHL, non-Hodgkin's lymphoma.

hospitalized at least once within 2 months after the visit at which they were screened compared with $23.5 \%$ of patients for whom overall protocol was not followed $\left(X^{2}=21.61 ; P=.000\right)$.

Table 3 presents the results of mixed-effects regression models analyzing associations of overall adherence with ED visits and hospitalizations. After controlling for the independent effects of institutional and patient characteristics on outcome, the risk ratio for ED use (0.82) indicates that overall adherence to a distress screening protocol is associated with an $18 \%$ reduction in the likelihood of using the 

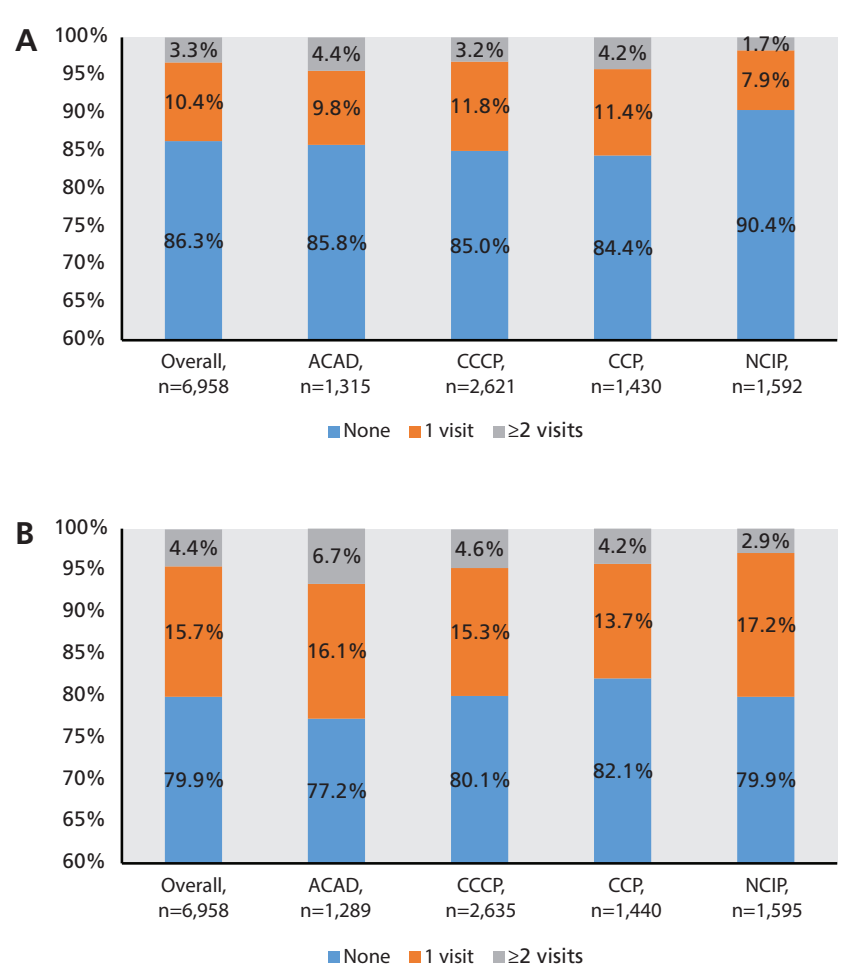

Figure 2. Service utilization within 2 months of screening date (frequency and percent) via (A) emergency department visits $(n=6,958)$ and (B) hospitalizations $(n=6,958)$.

Abbreviations: ACAD, Academic Comprehensive Cancer Program; CCCP, Comprehensive Community Cancer Program; CCP, Community Cancer Program; NCIP, NCl-designated Comprehensive Cancer Center Program.

ED. Similarly, the risk ratio for hospitalization (0.81) indicates that overall adherence was associated with a $19 \%$ reduction in likelihood for hospitalization.

\section{Discussion}

As of 2015, CoC Standard 3.2 requires demonstration of compliance with distress screening as a condition for cancer center accreditation. The $62.5 \%$ overall rate of adherence to a psychosocial distress screening protocol across a large and representative sample of cancer programs in the United States (and 2 in Canada) suggests that in the aggregate, cancer centers are approximately two-thirds of the way to full implementation of the standard. These findings are consistent with an earlier phase of the APAQCC evaluation study, in which cancer programs were characterized as doing moderately well in terms of organizational capacity to identify patients' psychosocial needs but less well in responding to those needs over time. ${ }^{26}$ Particularly concerning is the finding that documentation of psychosocial screen- ing is lacking in 1 of every 3 cases in this representative sample. The absence of these clinical data can compromise the ability of oncology care providers to know whether patients are receiving the psychosocial care and support they need when they need it.

The observed associations between overall adherence and medical service utilization are notable because they suggest opportunities for enhanced operational efficiencies and costs savings. In the current study, risks of using the ED and being hospitalized for those who were screened and responded to as per protocol were $18 \%$ to $19 \%$ less than those who were not screened and responded to according to protocol. Thus, if $100 \%$ of patients were screened and responded to according to their cancer program's protocol, then cancer programs that screen and respond to all their patients could expect, on average, $18 \%$ fewer ED visits and 19\% fewer hospitalizations when compared with settings in which there was no screening. This finding is consistent with a recent study in which the rate of ED visits among patients with breast cancer undergoing adjuvant chemotherapy was $43 \%$ lower for those screened for symptomatic distress compared with those not screened. ${ }^{33}$

Distress management protocols have the potential to uncover debilitating conditions or situations that, if properly identified and addressed, might prevent unnecessary use of scarce resources later. For example, distress screening could lead to identification of a patient's spouse/partner experiencing anxiety related to being responsible for maintaining the patient's central line at home. Appropriate screening and identification of distress would flag referral to a social worker, whose clinical assessment would uncover the cause of the patient's distress and lead to clinical engagement and delivery of an appropriate evidence-informed intervention (eg, cognitive behavioral therapy). In this instance, screening and appropriate response could preempt improper home care, reduce risk for central line infection, and thus prevent a subsequent patient visit to the ED or a hospitalization for septicemia, an oft-implicated reason for unplanned hospitalization among patients with cancer. ${ }^{34}$

Responding to $\mathrm{CoC}$ mandates for distress screening is challenging for cancer programs given the uneven distribution of resources and variations in institutional capacity to screen, assess, treat, and follow-up with all patients in need of services across the multiple and varied settings where patients receive 


\begin{tabular}{|c|c|c|}
\hline & $\begin{array}{l}\text { ED Use } \\
(95 \% \mathrm{Cl}) \\
(n=6,939)\end{array}$ & $\begin{array}{c}\text { Hospitalization } \\
(95 \% \mathrm{Cl}) \\
(\mathrm{n}=6,942)\end{array}$ \\
\hline Intercept & $0.08(0.049,0.133)^{* * *}$ & $0.122(0.082,0.182)^{* * *}$ \\
\hline \multicolumn{3}{|l|}{ Institution type } \\
\hline$A C A D$ & $1.88(1.135,3.099) *$ & $1.13(0.644,1.974)$ \\
\hline $\mathrm{CCCP}$ & $1.86(1.203,2.881) * *$ & $1.33(0.871,2.038)$ \\
\hline $\mathrm{CCP}$ & $1.98(1.194,3.298)^{* *}$ & $1.06(0.679,1.645)$ \\
\hline NCIP & Ref & Ref \\
\hline \multicolumn{3}{|l|}{ Minority cancer program } \\
\hline Yes, $\geq 40 \%$ racial minority patients & $1.21(0.825,1.771)$ & $1.20(0.808,1.776)$ \\
\hline No & Ref & Ref \\
\hline \multicolumn{3}{|l|}{ Age, $y$} \\
\hline $15-39$ & $0.96(0.692,1.320)$ & $0.87(0.681,1.103)$ \\
\hline $40-64$ & $0.93(0.812,1.063)$ & $1.11(1.004,1.219) *$ \\
\hline$\geq 65$ & Ref & Ref \\
\hline \multicolumn{3}{|l|}{ Race } \\
\hline White & $0.66(0.505,0.865)^{* *}$ & $0.93(0.794,1.095)$ \\
\hline Nonwhite & Ref & Ref \\
\hline \multicolumn{3}{|l|}{ Sex } \\
\hline Male & $1.00(0.832,1.212)$ & $1.03(0.898,1.170)$ \\
\hline Female & Ref & Ref \\
\hline \multicolumn{3}{|l|}{ Cancer type } \\
\hline Brain/CNS & $2.53(1.712,3.743)^{* * *}$ & $2.33(1.472,3.697)^{* * *}$ \\
\hline Hodgkin lymphoma & $1.34(0.574,3.106)$ & $0.83(0.326,2.136)$ \\
\hline $\mathrm{NHL}$ & $1.61(1.192,2.174)^{* *}$ & $1.65(1.245,2.198)^{* *}$ \\
\hline Leukemia & $1.05(0.614,1.785)$ & $1.39(0.912,2.124)$ \\
\hline Melanoma & $1.21(0.638,2.282)$ & $1.01(0.624,1.622)$ \\
\hline Colorectal & $1.72(1.286,2.302)^{* * *}$ & $1.60(1.231,2.067)^{* * *}$ \\
\hline All other gastrointestinal & $2.53(2.012,3.193) * * *$ & $1.88(1.494,2.355)^{* * *}$ \\
\hline Ovarian & $1.73(1.150,2.588) * *$ & $1.92(1.393,2.633)^{* * *}$ \\
\hline Prostate & $1.15(0.788,1.690)$ & $1.14(0.728,1.784)$ \\
\hline All other genitourinary & $1.37(0.952,1.959)$ & $1.93(1.420,2.620)^{* * *}$ \\
\hline Head and neck & $2.06(1.470,2.895)^{* * *}$ & $1.94(1.370,2.760)^{* * *}$ \\
\hline Lung & $2.35(1.889,2.933) * * *$ & $1.89(1.480,2.423)^{* * *}$ \\
\hline All other solid tumor/soft tissue & $1.93(1.302,2.870)^{* *}$ & $2.21(1.525,3.203)^{* * *}$ \\
\hline Breast (malignant) & Ref & Ref \\
\hline \multicolumn{3}{|l|}{ Overall adherence } \\
\hline Yes & $0.82(0.688,0.970)^{*}$ & $0.81(0.676,0.972) *$ \\
\hline No & Ref & Ref \\
\hline-2 log likelihood & $19,594.562$ & $19,649.614$ \\
\hline Random effects variance & 0.474 & 0.401 \\
\hline Intraclass coefficient - null model & 0.122 & 0.120 \\
\hline Variance accounted for by model & .262 & .105 \\
\hline
\end{tabular}

Generalized linear mixed effects models.

Abbreviations: ACAD, Academic Comprehensive Cancer Program; CCCP, Comprehensive Community Cancer Program; CCP, Community Cancer Program; CNS, central nervous system; ED, emergency department; NCIP, NCl-designated Comprehensive Cancer Center Program; NHL, nonHodgkin's lymphoma.

${ }^{*} P<.05 ; * * P<.01 ; * * * P<.001$. 
Distress Screening Adherence

care. ${ }^{26}$ Furthermore, the evidence base for these new patient-centered standards is underdeveloped and mixed in terms of demonstrating a positive effect of distress screening on patient outcomes and healthcare cost containment. ${ }^{1}$ Further research is needed to identify the patient, provider, and system characteristics that either hinder or promote implementation of comprehensive psychosocial care protocols, and to demonstrate whether these protocols improve the patient experience and contribute to reductions in medical service utilization and associated costs.

\section{Conclusions}

This study is the first large-scale empirical investigation of distress screening implementation in nonexperimental practice settings. Although limited in that neither a randomized controlled trial design nor standardized distress screening protocol was used across all participating treatment centers, the study is pragmatic and accounts for real-life conditions in which cancer treatment programs adapt distress screening protocols to match their own organizational structures, clinic operations, and available resources. The findings are not intended to suggest that screening adherence causes reductions in service utilization, because the study is limited by its cross-sectional design, modest rates of missing data (although comparable to studies using EHRs), lack of detailed patient data, and variation across participating cancer programs in terms of the complexity of cases treated. For example, a greater likelihood of ED use or hospital admission may be attributable to a patient's disease or treatment status. Alternatively, variations in rates of ED use and hospitalization attributed to lack of screening adherence may also be partially explained by the fact that larger academically affiliated cancer centers treat more complex cases and therefore patients may experience greater distress. Rates of ED use and hospitalization also may have been differentially affected in cases wherein an ED visit or hospital admission occurring outside the treating institution was not documented. However, the results derived from this substantially powered and sizeable study suggest that adherence to a standardized distress screening protocol is associated with, and may contribute to, enhanced operational efficiencies via reduction in use of medical services and their associated costs.

\section{Acknowledgments}

The authors wish to express their gratitude to Nina Miller, Krista Nelson, Sylvia Czisek, and Ryan McDougald for their assistance in preparing this manuscript, and to all of the APAQCC participants from the following cancer programs: Ann B. Barshinger Cancer Institute, Lancaster General Health, Lancaster, PA; Aspirus Regional Cancer Center, Wausau, WI; Athens Regional Medical Center, Athens, GA; Atlanta Cancer Care, Decatur, GA; Avera Cancer Institute, Sioux Falls, SD; Baptist Health Louisville Cancer Care Center, Louisville, KY; Beth Israel Deaconess Medical Center, Boston, MA; Bon Secours Richmond Health System, Midlothian, VA; Carolinas HealthCare System, Rutherford College, NC; Catawba Valley Medical Center, Hickory, NC; Central Maine Medical Center, Lewiston, ME; City of Hope National Medical Center, Duarte, CA; Duke Cancer Center, Durham, NC; Ellis Fischel Cancer Center/University of Missouri Health Care, Columbia, MO; Emory Saint Joseph's Hospital, Atlanta, GA; Cancer Center at Faxton St. Lukes Healthcare, Utica, NY; Good Samaritan Oncology/Hematology Associates, Lebanon, PA; GW Medical Faculty Associates, Washington, DC; Hannibal Regional Hospital/James E Cary Cancer Center, Hannibal, MO; Health Alliance Hospital of the Hudson Valley, Kingston, NY; Josephine Ford Cancer Institute, Henry Ford Health System, Detroit, MI; Huntsman Cancer Institute at the University of Utah, Salt Lake City, UT; Louis Stokes Cleveland VA Medical Center, Cleveland, OH; Mary Bird Perkins - Our Lady of the Lake Cancer Center, Baton Rouge, LA; Mayo Clinic Health System - Franciscan Healthcare, LaCrosse, WI; Mayo Clinic Health System Mankato, Mankato, MN; McLeod Regional Medical Center, Florence, SC; Medstar Georgetown University Hospital, Washington, DC; Memorial Regional Cancer Center, South Bend, IN; Mercy Hospital and Medical Center, Chicago, IL; Mercy Medical Center, Cedar Rapids, IA; MetroHealth Cancer Care Center, Cleveland, OH; Mount Sinai Hospital, Chicago, IL; Nash Cancer Treatment Center, Rocky Mount, NC; Northwestern Memorial Hospital, Chicago, IL; Oregon Health \& Science University Knight Cancer Institute, Portland, OR; Park Ridge Health, Hendersonville, NC; Presbyterian Healthcare Services, Albuquerque, NH; Princess Margaret Cancer Centre, Toronto, ON; Promedica Hickman Cancer Center, Sylvania, $\mathrm{OH}$; Providence Cancer Center, Portland, OR; Roger Williams Medical Center, Providence, RI; Sharp Memorial Hospital Outpatient Cancer Institute, San Diego, CA; St. Dominic Cancer Center, Jackson, MS; St. Joseph Comprehensive 
Zebrack et al

Cancer Center, Denver, CO; St. Jude Medical Center, Fullerton, CA; St. Luke's Hospital and Center for Cancer Care, Chesterfield, MO; St. Luke's Mountain States Tumor Institute, Boise, ID; St. Mary's Hospital Center, Montreal, Canada; Sutter Medical Center-Sutter Medical Foundation, Sacramento, CA; The Center for Cancer Prevention and Treatment - St. Joseph Hospital, Orange, CA; The Dale and Frances Hughes Cancer Center at Lehigh Valley Hospital-Pocono, East Stoudsburg, PA; The
DeCesaris Cancer Institute at Anne Arundel Medical Center, Annapolis, MD; McGlinn Cancer Institute, West Reading, PA; UC Davis Comprehensive Cancer Center, Sacramento, CA; UM Upper Chesapeake Medical Center, Kaufman Cancer Center, Bel Air, MD; University of Colorado Cancer Center, Aurora, CO; University of Kentucky Markey Cancer Center, Lexington, KY; Vermont Cancer Center/Fletcher Allen Health Care, Burlington, VT; Wellspan York Cancer Center, York, PA.

\section{References}

1. Carlson LE, Waller A, Mitchell AJ. Screening for distress and unmet needs in patients with cancer: review and recommendations. J Clin Oncol 2012;30:1160-1177.

2. Carlson LE, Angen M, Cullum J, et al. High levels of untreated distress and fatigue in cancer patients. Br J Cancer 2004;90:2297-2304.

3. Zabora JR, BrintzenhofeSzoc K, Curbow B, et al. The prevalence of psychological distress by cancer site. Psychooncology 2001;10:19-28.

4. Zabora JR, Blanchard CG, Smith ED, et al. Prevalence of psychological distress among cancer patients across the disease continuum. J Psychosoc Oncol 1997;15:73-87.

5. Gao W, Bennett M, Stark D, et al. Psychological distress in cancer from survivorship to end of life care: prevalence, associated factors and clinical implications. Eur J Cancer 2010;46:2036-2044.

6. Zabora JR, MacMurray L. The history of psychosocial screening among cancer patients. J Psychosoc Oncol 2012;30:625-635.

7. Holland JC, Jacobsen PB, Andersen B, et al. NCCN Clinical Practice Guidelines in Oncology for Distress Management, Version 2.2016. Accessed February 9, 2017. To view the most recent version of these guidelines, visit NCCN.org.

8. Pirl WF, Jacobsen PB, DeShields TL. Opportunities for improving psychosocial care for cancer survivors. J Clin Oncol 2013;31:1920-1921.

9. Barrera I, Spiegel D. Review of psychotherapeutic interventions on depression in cancer patients and their impact on disease progression. Int Rev Psychiatry 2014;26:31-43.

10. Han X, Lin CC, Li C, et al. Association between serious psychological distress and health care use and expenditures by cancer history. Cancer 2015;121:614-622.

11. Hollingworth W, Metcalfe $C$, Mancero S, et al. Are needs assessments cost effective in reducing distress among patients with cancer? A randomized controlled trial using the distress thermometer and problem list. J Clin Oncol 2012;31:3631-3638.

12. Carlson LE. Screening alone is not enough: the importance of appropriate triage, referral, and evidence-based treatment of distress and common problems. J Clin Oncol 2013;31:3616-3617.

13. Institute of Medicine. Cancer Care for the Whole Patient: Meeting Psychosocial Health Needs. Washington, DC: The National Academies Press; 2008.

14. Commission on Cancer. Cancer Program Standards: Ensuring PatientCentered Care. Available at: https://www.facs.org/quality-programs/ cancer/coc/standards. Accessed February 9. 2017.

15. Chiang AC, Amport SB, Corjulo D, et al. Incorporating patient-reported outcomes to improve emotional distress screening and assessment in an ambulatory oncology clinic. J Oncol Pract 2015;11:219-222.

16. Carlson LE, Groff SL, Maciejewski O, Bultz BD. Screening for distress in lung and breast cancer outpatients: a randomized controlled trial. J Clin Oncol 2010;28:4884-4891.

17. Coyne JC. Benefits of screening cancer patients for distress still not demonstrated. Br J Cancer 2013;108:736-737.
18. Meijer A, Roseman M, Delisle VC, et al. Effects of screening for psychological distress on patient outcomes in cancer: a systematic review. J Psychosom Res 2013;75:1-17.

19. Pirl WF, Lennes IT. Quality of psychosocial services in cancer centers: today and tomorrow. Cancer 2016;122:1807-1808.

20. Zebrack B, Burg M, Vaitones V. Distress screening: an opportunity for enhancing quality cancer care and promoting the oncology social work profession. J Psychosoc Oncol 2012;30:615-624.

21. Faller $H$, Schuler $M$, Richard $M$, et all. Effects of psycho-oncologic interventions on emotional distress and quality of life in adult patients with cancer: systematic review and meta-analysis. J Clin Oncol 2013;31:782-793.

22. Schneider S, Moyer A, Knapp-Oliver S, et al. Pre-intervention distress moderates the efficacy of psychosocial treatment for cancer patients: a meta-analysis. J Behav Med 2010;33:1-14.

23. Meyer TJ, Mark MM. Effects of psychosocial interventions with adult cancer patients: a meta-analysis of randomized experiments. Health Psychol 1995;14:101-108.

24. Cwikel J, Behar L, Rabson-Hare J. A comparison of a vote count and meta-analysis review of intervention research with adult cancer patients. Res Soc Work Pract 2000;10:139-158.

25. McQuellon RP, Danhauer SC. Psychosocial rehabilitation in cancer care. In: Chang AE, Ganz PA, Hayes DF, et al, eds. Oncology: An Evidence Based Approach. New York, NY: Springer; 2006:1942-1954.

26. Zebrack B, Kayser K, Padgett LS, et al. Institutional capacity to provide psychosocial oncology support services: a report from the Association of Oncology Social Work. Cancer 2016;122:1937-1945.

27. McCaskill-Stevens W, McKinney MM, Whitman CG, Minasian LM. Increasing minority participation in cancer clinical trials: the minority based community clinical oncology program experience. J Clin Oncol 2005;23:5247-5254.

28. Raudenbush SW, Bryk AS. Hierarchical Linear Models: Applications and Data Analysis Methods. Thousand Oaks, CA: Sage Publications; 2002.

29. Powers DA, Xie Y. Statistical Methods for Categoriecal Data Analysis. 2nd ed. Bingley, UK: Emerald Group Publishing; 2008.

30. Madden JM, Lakoma MD, Rusinak D, et al. Missing clinical and behavioral health data in large electronic health record (EHR) systems. J Am Med Inform Assoc 2016;23:1143-1149.

31. Berger ML, Curtis MD, Smith G, et al. Opportunities and challenges in leveraging electronic health record data in oncology. Future Oncol 2016;12:1261-1274.

32. Enders CK. Applied Missing Data Analysis. New York, NY: Guilford; 2010.

33. Barbera L, Sutradhar R, Howell D, et al. Does routine symptom screening with ESAS decrease ED visits in breast cancer patients undergoing adjuvant chemotherapy? Support Care Cancer 2015;23:3025-3032.

34. Manzano J, Luo R, Elting LS, Suarez-Almazor M. Unplanned hospitalizations among gastrointestinal cancer patients in the state of Texas [abstract]. J Clin Oncol 2013;31(Suppl):Abstract 6634. 